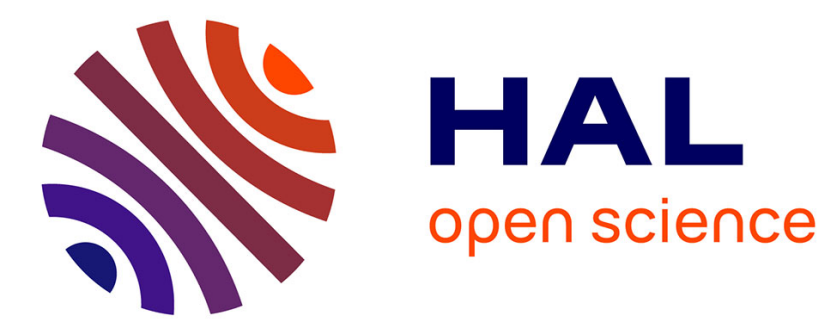

\title{
Thermal-light full-field optical coherence tomography in the 1.2 micron wavelength region
}

\author{
Arnaud Dubois, Gael Moneron, Claude Boccara
}

\section{To cite this version:}

Arnaud Dubois, Gael Moneron, Claude Boccara. Thermal-light full-field optical coherence tomography in the 1.2 micron wavelength region. Optics Communications, 2006, 266, pp.738. 10.1016/j.optcom.2006.05.016 . hal-00520541

\section{HAL Id: hal-00520541 \\ https://hal.science/hal-00520541}

Submitted on 24 Jan 2012

HAL is a multi-disciplinary open access archive for the deposit and dissemination of scientific research documents, whether they are published or not. The documents may come from teaching and research institutions in France or abroad, or from public or private research centers.
L'archive ouverte pluridisciplinaire HAL, est destinée au dépôt et à la diffusion de documents scientifiques de niveau recherche, publiés ou non, émanant des établissements d'enseignement et de recherche français ou étrangers, des laboratoires publics ou privés. 


\title{
Thermal-light full-field optical coherence tomography in the $1.2 \mu \mathrm{m}$ wavelength region
}

\author{
A. Dubois, G. Moneron and A.C. Boccara \\ Laboratoire d'Optique Physique, \\ Ecole Supérieure de Physique et Chimie Industrielles, \\ Centre National de la Recherche Scientifique, UPR A0005, \\ 10 rue Vauquelin, F-75231 Paris Cedex 5, France
}

\begin{abstract}
Author Keywords: Optical coherence tomography; Biomedical imaging; Optical tomography; Microscopy; Infrared camera

PACS classification codes: $42.25 . \mathrm{Hz}$ Interference; 42.25.Kb coherence; 42.30-d Imaging and optical processing
\end{abstract}

\begin{abstract}
Full-field optical coherence tomography with dynamic focusing correction is demonstrated in the $1.2 \mu \mathrm{m}$ wavelength region using an Indium Gallium Arsenide area camera as image sensor and a halogen lamp as illumination source. An axial image resolution of $1.6 \mu \mathrm{m}$ is measured in air ( $1.1 \mu \mathrm{m}$ in tissue) which is the best resolution ever achieved in OCT in this wavelength region. En face tomographic images are produced using a phase-shifting method requiring the acquisition of only two phase-opposed interferometric images. In spite of a relatively moderate detection sensitivity of $80 \mathrm{~dB}$ within $1.0 \mathrm{~s}$ image acquisition time, ultrahigh resolution imaging in highly scattering biological tissues is possible without immersion medium.
\end{abstract}




\section{Introduction}

Optical coherence tomography (OCT) is an emerging imaging technique which provides information on biological tissue morphology [1-3]. OCT has the potential to replace conventional biopsy and histopathology, provided that millimeter-range penetration depth and micrometer-scale resolution are possible.

OCT penetration depth depends on scattering and absorption of light within the biological tissues. The intensity of light scattered by particles varies with the optical wavelength $\lambda$ as $1 / \lambda^{k}$, where $\mathrm{k}>0$ is a parameter which depends on the particle size, geometry and refractive index [4]. Light scattering in biological tissues cannot be easily modeled but it obviously becomes weaker as wavelength is increased [5-7]. The absorption of light in tissue is dominated by the absorption by water, since water is the main constituent of biological media. The absorption by water is minimal at the wavelength of $\lambda \sim 0.5 \mu \mathrm{m}$ and has a general trend to increase with wavelength as shown in Fig. 1 [8]. The optimal wavelength to maximize the OCT imaging penetration results from the best trade-off between absorption and scattering. Previous works have shown better penetration depth in highly scattering tissues for OCT operating in the $1.3 \mu \mathrm{m}$ wavelength region compared to $0.8 \mu \mathrm{m}[7]$.

The axial resolution of OCT is proportional to $\lambda_{0}^{2} / \Delta \lambda$, where $\lambda_{0}$ is the center wavelength and $\Delta \lambda$ is the spectral width of the light source [3]. Increasing the center wavelength thus requires increasing the spectral width in a quadratic manner in order to maintain the axial resolution. Efforts have been concentrated on the development of broad-bandwidth light sources in the $1.3 \mu \mathrm{m}$ wavelength region that would permit OCT micrometer-scale resolution $[9,10]$. Recently, $1.4 \mu \mathrm{m}$ axial resolution in ex vivo human skin and arterial biopsies has been demonstrated using a broad-bandwidth light source based on a pulsed erbium fiber laser coupled with a highly nonlinear fiber to generate a supercontinuum in the $1300 \mathrm{~nm}$ wavelength region [11]. 
Full-field OCT is an original approach of OCT, which uses spatially and temporally incoherent light to illuminate the whole field to be imaged [12-15]. In contrast with conventional OCT, full-field OCT provides tomographic images in the en face (transverse) orientation without scanning of a light beam. The tomographic images are obtained by combination of interferometric images acquired with an image sensor such as a CCD (charge coupled device) digital camera. Full-field OCT, sometimes referred to as full-field optical coherence microscopy, is based on an interference microscope illuminated with a simple white-light lamp. Due to the ultra-short coherence length of this illumination source, the axial resolution is better than that of conventional OCT. The transverse resolution of full-field OCT is similar to that of microscopy, which is better than the one of OCT producing axially-oriented tomographic images. We recently demonstrated an ultrahigh resolution of $0.7 \mu \mathrm{m} \times 1.0 \mu \mathrm{m}$ (axial $\times$ transverse) in tissues with fullfield OCT at $\sim 0.8 \mu \mathrm{m}$ center wavelength using a halogen lamp and a silicon-based CCD camera [16].

In this paper we present a thermal-light full-field OCT system operating in the $1.2 \mu \mathrm{m}$ wavelength region where the imaging depth in tissue is presumably greater owing to lower scattering. This new system uses an Indium Gallium Arsenide (InGaAs) area camera and a simplified method for faster image acquisition. We mention that this kind of camera has already been used for full-field OCT imaging with a mode-locked Cr4+:forsterite laser [17]. The spatial resolution and detection sensitivity were however relatively weak. We demonstrate here ultrahigh spatial resolution full-field OCT without immersion medium thanks to dynamic focusing adjustments. Numerical simulations have been performed to quantify the effect of water dispersion and absorption on the axial resolution. The performances of the instrument are reported. Tomographic images relatively deep inside highly scattering tissue such as mouse skin are presented. 


\section{Principle}

\section{Experimental set-up}

The principle of full-field OCT relies on low coherence interference microscopy. The experimental arrangement is based on a bulk Michelson interferometer with identical microscope objectives in both arms, as depicted in Fig. 2. This configuration is referred to as the Linnik interferometer $[14,18,19]$. An easy way to produce light with extremely broad and smooth spectrum with adjustable center wavelength is to use a thermal light source [20]. The black body radiation spectrum is given by Planck's law [21]:

$I(\lambda)=\frac{2 h c^{2}}{\lambda^{5}} \frac{1}{\exp (h c / \lambda k T)-1}$,

where $T$ is the temperature of the black body, $h$ the Planck's constant, $c$ the speed of light, and $k$ the Boltzmann's constant. At the temperature of $2250 \mathrm{~K}$, the maximum of the black body radiation spectrum is at $\lambda=1.3 \mu \mathrm{m}$ with a full width at half maximum (FWHM) of $\Delta \lambda=$ $1.55 \mu \mathrm{m}$. A tungsten halogen lamp is not truly a black body. Its spectrum is however very broad and a lot of infrared light can be emitted. The drawback of a thermal light source such as a halogen lamp is its low brightness. As a consequence it cannot be used for scanning OCT. Nevertheless, the low spatial coherence of a thermal source is a great advantage for full-field imaging. A halogen lamp is indeed particularly appropriate for full-field OCT in the near infrared.

A halogen lamp (12V-100W), with a stabilized DC power supply, is incorporated in a classical Köhler system for homogeneous illumination of the whole microscope objective fields. Light is split into the reference and sample arms by a broadband beam-splitter. After recombination by the beam splitter, the sample and reference images are projected onto an image sensor using an achromatic doublet lens optimized around $1.3 \mu \mathrm{m}$. The image sensor is an Indium Gallium Arsenide (InGaAs) area camera (model SU320MS-1.7RT Snapshot commercialized by Sensors Unlimited, Inc). The camera has $320 \times 256$ pixels with $25 \mu \mathrm{m}$ pitch, delivers $100 \%$ fill factor and has $>70 \%$ quantum efficiency from $1.0 \mu \mathrm{m}$ to $1.6 \mu \mathrm{m}$. The camera 
frame rate is $50 \mathrm{~Hz}$. Dry microscope objectives are used without immersion media because of the absorption of infrared light by water. The reference mirror is a piece of glass providing $4 \%$ reflectivity. The objective in the object arm is mounted on a motorized translation stage for dynamic focusing adjustments as will be explained later. The object can be translated in the axial direction using another motorized translation stage in order to image at different depths.

\section{Image acquisition}

Due to the low temporal coherence of the illumination source, interference occurs only when the optical path lengths of the two interferometer arms are nearly identical. The interference fringes are comprised in a narrow envelop with width equal to the coherence length of the source. When a biological object is placed under the microscope objective in the object arm, the light reflected by the reference mirror interferes with the light reflected or backscattered by the sample structures contained in a limited volume (the "coherence volume"). This volume is a slice orthogonal to the objective axis, located at a depth inside the object defined by an optical path length difference of zero (this depth defines the "coherence plane"). The thickness of the slice is determined by the width of the fringe envelop. The extraction of the fringe envelop yields therefore an en face tomographic image.

The fringe envelope is extracted using a simple phase-shifting method to optimize the operation speed. A high acquisition speed is of particular importance for imaging of biological objects which cannot remain immobile during long times. Two phase-opposed interferometric images are produced by making the reference mirror oscillating between two positions distant of a quarter of the illumination center wavelength (i.e. $\sim 0.3 \mu \mathrm{m}$ ). The photon flux on each pixel of the CCD (coordinates $(x, y)$ ) can be written for the two reference mirror positions as

$$
\left\{\begin{array}{l}
I_{1}(x, y)=\frac{I_{0}}{4}\left[R_{i n c}(x, y)+R_{r e f}+2 \sqrt{R_{o b j}(x, y) R_{r e f}} \cos \phi(x, y)\right] \\
I_{2}(x, y)=\frac{I_{0}}{4}\left[R_{i n c}(x, y)+R_{r e f}-2 \sqrt{R_{o b j}(x, y) R_{r e f}} \cos \phi(x, y)\right]
\end{array},\right.
$$


where $\phi(x, y)$ represents the optical phase and $I_{0}$ is the photon flux at the entrance of the interferometer. $R_{r e f}$ is the (uniform) reference mirror reflectivity, $R_{o b j}(x, y)$ is the proportion of light reflected by the object which interferes and $R_{i n c}(x, y)$ the proportion of light reflected by the object and collected by the microscope objective, which does not interfere. In other words, $R_{o b j}(x, y)$ represents the reflectivity distribution of the sample structures contained in the coherence volume. $R_{o b j}(x, y)$ thus corresponds to an en face tomographic image. The camera acquires a number $N$ of image pairs. The images corresponding to the same reference mirror positions are summed. The two resulting images $\left(E_{1}(x, y), E_{2}(x, y)\right)$ correspond to $N$ times the integration of the photon flux $\left(I_{1}(x, y), I_{2}(x, y)\right)$ over the camera exposure time $T$. We denote by $\eta$ the quantum efficiency of the camera. The total number of photoelectrons delivered by each camera pixel is

$$
E_{1,2}(x, y)=\eta N \int_{0}^{T} I_{1,2}(x, y) d t
$$

To maximize the signal-to-noise ratio, the illumination flux is adjusted so that the camera pixel wells are close to saturation. The number of photoelectrons produced by each pixel is then close to the full-well capacity $\xi_{\text {sat. }}$. Since $R_{o b j}(x, y)<<R_{r e f}$, we can write according to Eq. (2) that

$$
\xi_{\text {sat }} \approx \frac{\eta I_{0} T}{4}\left(R_{r e f}+R_{i n c}\right)
$$

By combining the previous equations, we obtain the following relation:

$$
\left(E_{1}-E_{2}\right)^{2}=8\left(N \xi_{\text {sat }}\right)^{2} \frac{R_{r e f}}{\left(R_{r e f}+R_{i n c}\right)^{2}} R_{o b j} \cos ^{2} \phi
$$

The en face tomographic image, $R_{o b j}(x, y)$, can be thus calculated from two phase-opposed interferometric images as expressed in Eq. (5). Actually, the fringe envelope is not truly extracted since the phase $\phi(x, y)$ is not eliminated. Due to the size and distribution of the structures in most biological media, the fringes are generally not visible in the images. The most 
important function of signal acquisition in OCT is to eliminate the contribution of the light which has not interfered. This function is perfectly accomplished by the method presented here. A home-written Visual $\mathrm{C}++$ software was developed to calculate and display the tomographic image in real-time (frame rate of $1 \mathrm{~Hz}$ for $2 \times 25$ accumulated images).

\section{Dynamic focusing adjustments}

As the object is translated in the axial direction to obtain en face tomographic images deeper and deeper, the coherence plane and the focal plane move away from each other because the optical path in air is progressively replaced by an optical path in the biological tissues with higher refractive index (see Fig. 3). Note that this phenomenon is very weak when water immersion microscope objectives are used since biological tissues have refractive index close to that of water [22].

In a Linnik interference microscope the contrast of the interferometric signal is reduced if one of the objectives is not perfectly in focus [14]. For this reason, the coherence plane must coincide with the focal plane of the objectives in full-field OCT, otherwise the tomographic images vanishes. Focusing adjustments are therefore required as the object is moved up. Using the Snell's law at third order in incidence angle $\alpha$, we calculate that in order to image at a depth $\mathrm{z}$ below the surface of the object with refractive index $n$, the object must be elevated of a distance equal to $\mathrm{n} \times \mathrm{z}$, while the objective in the object arm must be elevated of a distance

$Z=\left(\frac{n^{2}-1}{n}\right) z$

Two computer-controlled motorized translation stages are used to move up both the object and the objective (see Fig. 1). 


\section{Performances}

\section{Spatial resolution}

The axial resolution of our full-field OCT system is governed by the effective optical spectrum, which is given by the product of the halogen lamp spectrum, the spectral response of the camera and the spectral transmission of the system optical components. We measured the interferogram response of the system in air using a silver mirror as object (see Fig. 4 a). The effective spectrum was deduced from Fourier transform of the interferogram (see Fig. 4 b). The center wavelength was found to be at $\lambda=1.2 \mu \mathrm{m}$ and the FWHM of the fringe envelope to be $1.6 \mu \mathrm{m}$. Assuming a tissue refractive index of 1.4 , the axial resolution for superficial tissue imaging is therefore $\sim 1.1 \mu \mathrm{m}$. This is the best axial resolution ever achieved in OCT in this wavelength region. If the optical dispersion is not equal in the two interferometer arms, the axial resolution is degraded [23]. We have performed numerical simulations including the effect of water dispersion and absorption to quantify the degradation of axial resolution when imaging in water, the reference arm being in air [24]. The actual effective spectrum (shown in Fig. 4 b) is considered in the calculations. According to these simulations the degradation of the axial resolution is only of a factor of 1.25 at a depth of $500 \mu \mathrm{m}$ (see Fig. 5). Dynamic compensation of dispersion is therefore not required in this system. We simply placed fixed antireflection-coated glass plates in both arms to compensate for dispersion mismatch introduced by the microscope objectives which are not perfectly identical (see Fig. 2)

In principle, the transverse resolution is imposed by the numerical aperture of the objectives and the illumination wavelength. Because the objectives are designed for visible light, optical aberrations however degrade the transverse resolution. By imaging the edge of a silicon wafer we estimated the transverse resolution to be $\sim 3 \mu \mathrm{m}$ in air [25-27]. 


\section{Detection sensitivity}

Detection sensitivity is a crucial parameter in OCT, because it determines the image quality and the maximum depth to which imaging is possible. The detection sensitivity of full-field OCT depends essentially on the charge capacity of the camera pixels [14-16]. The InGaAs camera has a large pixel full well capacity of $\xi_{\text {sat }}=8 \times 10^{5}$ electrons. Assuming the measurements to be shotnoise limited, the tomographic image would then be calculated as $\left[\left(E_{1}+v_{1}\right)-\left(E_{2}+v_{2}\right)\right]^{2}$, where $v$ is an additive noise with the following properties:

$$
\left\{\begin{array}{l}
\left\langle v_{1}\right\rangle=\left\langle v_{2}\right\rangle=\left\langle v_{1} v_{2}\right\rangle=0 \\
\left\langle v_{1}^{2}\right\rangle=\left\langle v_{2}^{2}\right\rangle=\sigma^{2}
\end{array} .\right.
$$

The angle brackets in Eq. (7) denote a time average. We assume that the shot-noise results from the fundamental photon noise. According to the Poisson statistic, the shot-noise variance $\sigma^{2}$ is then equal to the number of photons detected by each camera pixel multiplied by their quantum efficiency. Since the illumination flux is adjusted so that the well of each camera pixel is close to saturation and $N$ images are accumulated, we have $\sigma^{2} \approx N \xi_{\text {sat }}$. The background noise in our imaging instrument can be regarded as the average value of a tomographic image when there is no interference (i.e. when $\left.E_{1}=E_{2}\right)$. This background noise is equal to $\left\langle\left(v_{1}-v_{2}\right)^{2}\right\rangle=2 N \xi_{\text {sat }}$. The signal-to-noise ratio in an image can thus be written as

$$
\mathrm{SNR}=\frac{\left(E_{1}-E_{2}\right)^{2}}{2 N \xi_{s a t}}
$$

Considering that the minimum detectable reflectivity corresponds to a signal-to-noise ratio equal to 1 we establish, using Eq. (8) and Eq. (5) with $\cos ^{2} \phi=1 / 2$ (in average over the image), that the smallest detectable reflectivity $R_{o b j}=R_{\min }$ is 
$R_{\text {min }}=\frac{\left(R_{r e f}+R_{i n c}\right)^{2}}{2 N \xi_{s a t} R_{r e f}}$.

Theoretical values of $R_{\min }$ as a function of the number $N$ of image accumulation are plotted in Fig. 6.

Measurements of the detection sensitivity are also reported in Fig. 6. The measured sensitivity is $\sim 10 \mathrm{~dB}$ above the theoretical shot-noise resulting from the ultimate photon noise. This difference is due to a significant electrical noise of the InGaAs camera. Accumulating images yields to an increase of the detection sensitivity. In practice, we accumulate $2 \times 25$ images, which represents an acquisition time of $1.0 \mathrm{~s}$. A detection sensitivity of $80 \mathrm{~dB}$ is then reached.

\section{Image example}

To illustrate the performances of the new full-field OCT system presented in this paper, we imaged skin of a mouse ear ex vivo. Figure 6 shows a vertical (XZ) tomographic image reconstructed from a stack of en face tomographic images acquired with steps of $1 \mu \mathrm{m}$. In spite of the moderate detection sensitivity, we can image through the entire thickness of the ear $(\sim 300 \mu \mathrm{m})$. These results shows that the illumination wavelength in the $1.2 \mu \mathrm{m}$ region is appropriate for imaging inside highly scattering biological media.

\section{Conclusion}

In summary, we have developed a full-field OCT system with dynamic focusing correction operating in the $1.2 \mu \mathrm{m}$ wavelength region. By use of a simple halogen lamp, the best resolution ever achieved by OCT at this wavelength is obtained. The system uses dry microscope objectives, which is of great interest for applications where immersion media cannot be used. 
The image field is currently limited by the low number of pixels of the camera. With the development of high resolution InGaAs area cameras, this limitation will probably be overcome in a near future. Moreover, high-resolution high-speed cameras would enable pixel binning to improve the detection sensitivity while maintaining short image acquisition times. 


\section{References}

1. D. Huang, E.A. Swanson, C.P. Lin, J.S. Schuman, W.G. Stinson, W. Chang, M.R. Hee, T. Flotte, K. Gregory, C.A. Puliafito, J.G. Fujimoto, Science 254 (1991) 1178-1181.

2. J.G. Fujimoto, M.E. Brezinski, G.J. Tearney et al, Nature Med. 1 (1995) 970-972.

3. A.F. Fercher, J. Biomed. Opt. 1 (1996) 157-173.

4. J. Schmitt, S.H. Xiang, K.M. Yung, J. Opt. Soc. Am. A 15 (1998) 2288-2296.

5. R.R. Anderson and J.A. Parrish, J. Invest. Dermatol. 77 (1981) 13-19.

6. P. Parsa, S.L. Jacques, and N.S. Nishioka, Appl. Opt. 28 (1989) 2325-2330.

7. J. M. Schmitt, A. Knuttel, M. Yadlowsky, M.A. Eckhaus, Phys. Med. Biol. 39 (1994) 17051720.

8. G.M. Hale and M.R. Querry, Appl. Opt. 12 (1973) 555-563.

9. C. Chudoba, J.G. Fujimoto, E.P. Ippen, H.A. Haus, U. Morgner, F.X. Kärtner, V. Scheuer, G. Angelov, T. Tschudi, Opt. Lett. 26 (2001) 292-294.

10. I. Hartl, X.D. Li, C. Chudoba, R.K. Ghanta, T.H. Ko, J.G. Fujimoto, Opt. Lett. 26 (2001) 608-610.

11. K. Bizheva, B. Považay, B. Hermann, H. Sattmann, W. Drexler, M. Mei, R. Holzwarth, T. Hoelzenbein, V. Wacheck, H. Pehamberger, Opt. Lett. 28 (2003) 707-709.

12. E. Beaurepaire, A.C. Boccara, M. Lebec, L. Blanchot, and H. Saint-Jalmes, Opt. Lett. 23 (1998) 244-246.

13. L. Vabre, A. Dubois, A.C. Boccara, Opt. Lett. 27 (2002), 530-532.

14. A. Dubois, L. Vabre, A.C. Boccara, and E. Beaurepaire, Appl. Opt. 41 (2002) 805-812.

15. B. Laude, A. De Martino, B. Drevillon, L. Benattar, and L. Schwartz, Appl. Opt. 41 (2002) 6637-6645.

16. A. Dubois, K. Grieve, G. Moneron, R. Lecaque, L. Vabre, A.C. Boccara, Appl. Opt. 43 (2004) 2874-2883.

17. E. Bordenave, E. Abraham, G. Jonusauskas, N. Tsurumachi, J. Oberle, C. Rulliere, P.E. Minot, M. Lassegues, J.E. Surleve Bazeille, Appl. Opt. 41 (2002) 2059-2064.

18. M. Davidson, K. Kaufman, I. Mazor, F. Cohen, in: Integrated Circuit Metrology, Inspection, and Process Control, K. M. Monahan (Ed.), Proc. Soc. Photo-Opt. Instrum. Eng. 775 (1987) 233-247.

19. G.S. Kino, S.C. Chim, Appl. Opt. 29 (1990) 3775-3783.

20. A.F. Fercher, C.K. Hitzenberger, M. Sticker, E. Moreno-Barriuso, R. Leitgeb, W. Drexler, H. Sattmann, Opt. Commun. 185 (2000) 57-64.

21. W. Heitler, in: Courier Dover Publications (Ed.), The Quantum Theory of Radiation 1984.

22. J. Lai, Z. Li, C. Wang, and A. He, Appl. Opt. 44 (2005) 1845-1849.

23. C.K. Hitzenberger, A. Baumgartner, W. Drexler, A.F. Fercher, J. Biomed. Opt. 4 (1999) 144151.

24. T.R. Hillman, D.D. Sampson, Opt. Express 13 (2005) 1860-1874.

25. S.M. Bentzen, Med. Phy. 10 (1983) 579-581.

26. D.J. Hall, J.C. Hebden, D.T. Delpy, Med. Phys. 24 (1997) 361-368.

27. A. Dubois, A.C. Boccara, M. Lebec, Opt. Lett. 24 (1999) 309-311 


\section{Figure captions}

Fig.1: Optical absorption of water [data from Reference 8].

Fig. 2. Experimental arrangement. BS: beam splitter (broadband, non polarizing), MO:

microscope objective (10x, 0.25 NA), L: aplanetic doublet achromat lenses (500 mm focal length, optimized around $1.3 \mu \mathrm{m}$ ). The reference mirror is a piece of glass ( $4 \%$ reflectivity). The InGaAs area camera $(320 \times 256$ pixels $)$ operates at $50 \mathrm{~Hz}$. DCP: dispersion compensation glass plates. The filter eliminates wavelengths below $750 \mathrm{~nm}$.

Fig. 3. The interferometer is adjusted so that the focal plane and coherence plane coincide at the surface of the object (A). When the object is elevated of a distance e, the coherence plane is then located at e/n below the object surface, and the focal plane at $\mathrm{n} \times \mathrm{e}(\mathrm{B})$. The focal plane matches the coherence plane again if the objective is elevated of a distance $e\left(1-1 / \mathrm{n}^{2}\right)(C)$.

Fig. 4. Interferogram response of the system measured in air (a). The interferogram envelop has a full width at half maximum of $1.6 \mu \mathrm{m}$. The effective spectrum of the system (b) is calculated by Fourier transform of the interferogram. The center wavelength is measured to be of $1.2 \mu \mathrm{m}$.

Fig. 5. Calculation of the axial resolution as a function of depth when imaging in water, the reference arm being in air. The axial resolution is defined as the full width at half maximum of the interferogram envelop. The insert shows the interferogram at $500 \mu \mathrm{m}$ depth. The experimental illumination spectrum (shown in Fig. 4b) is used for these numerical simulations taking into account the effect of both dispersion and absorption by water.

Fig. 6. Detection sensitivity as a function of image accumulation. The theoretical sensitivity is calculated using Eq. (9) with $R_{\text {ref }}=0.04, R_{\text {inc }}=0.01, \xi_{\text {sat }}=8 \times 10^{5}$.

Fig. 7. Vertical (XZ) tomographic image of mouse ear skin (ex vivo) obtained with full-field OCT in the $1.2 \mu \mathrm{m}$ wavelength region. 


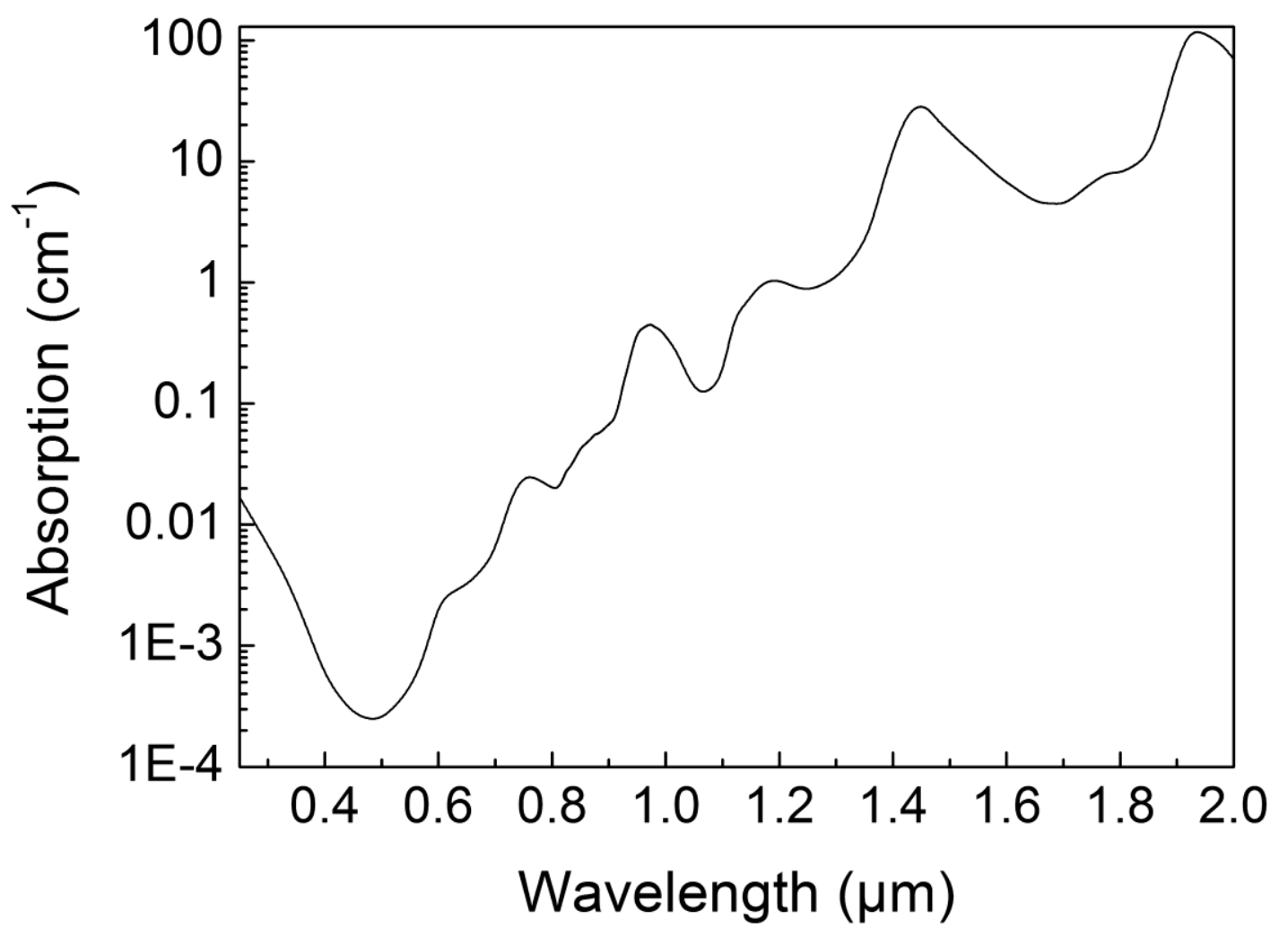

Fig. 1 


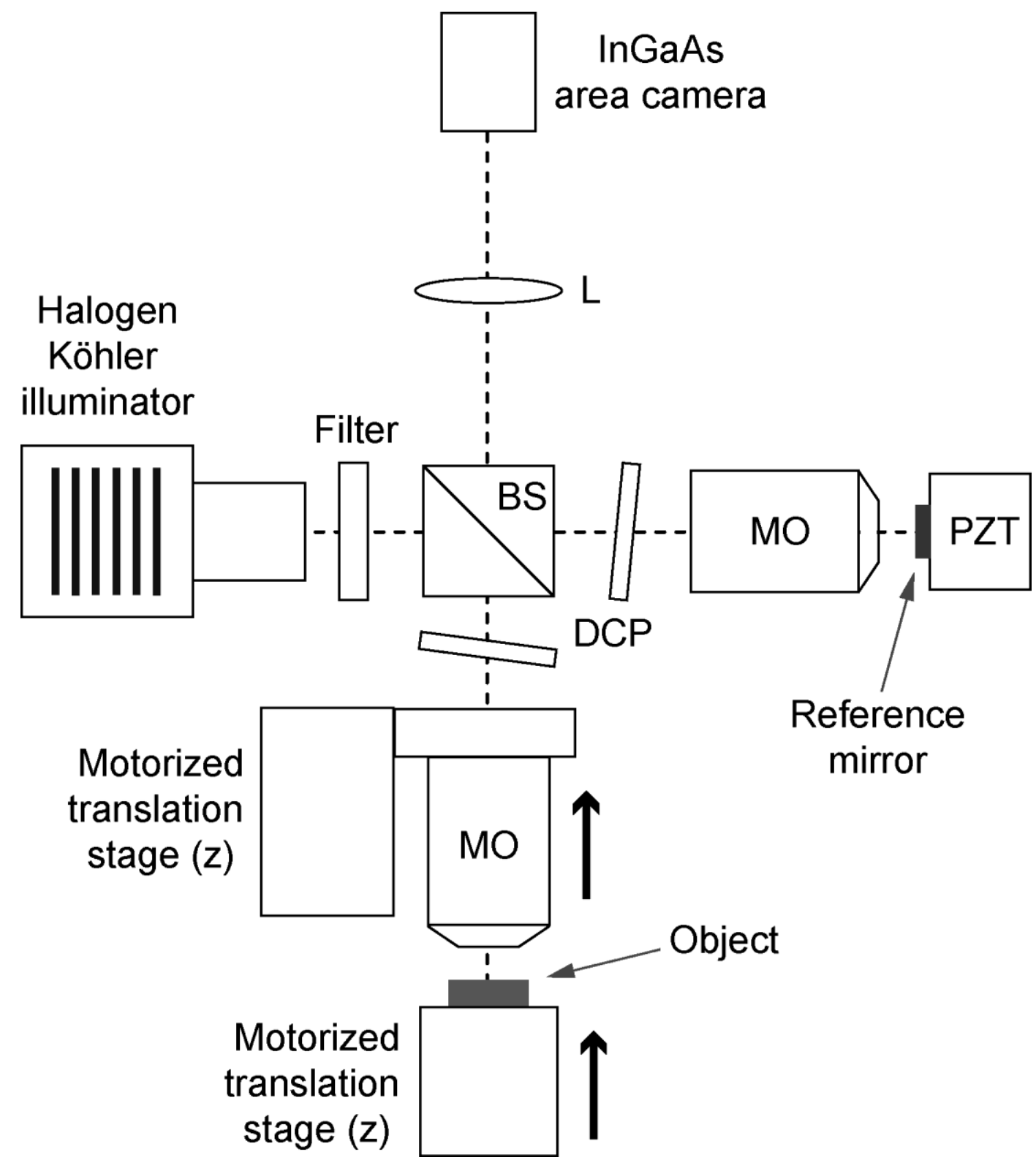

Fig. 2 


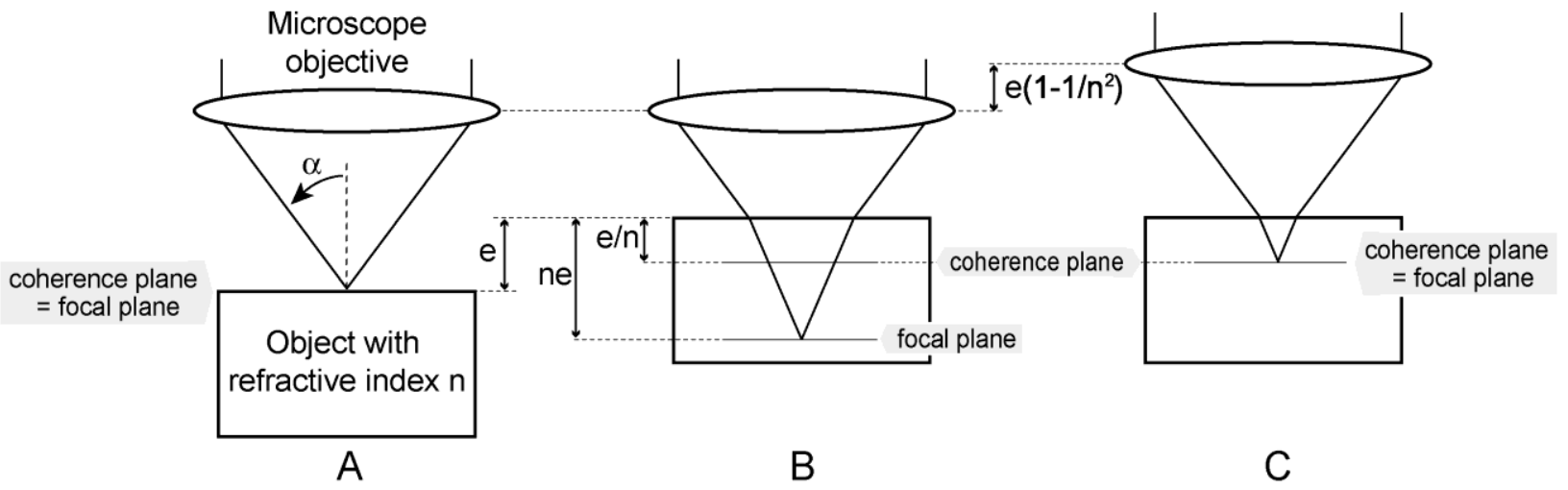

Fig. 3 

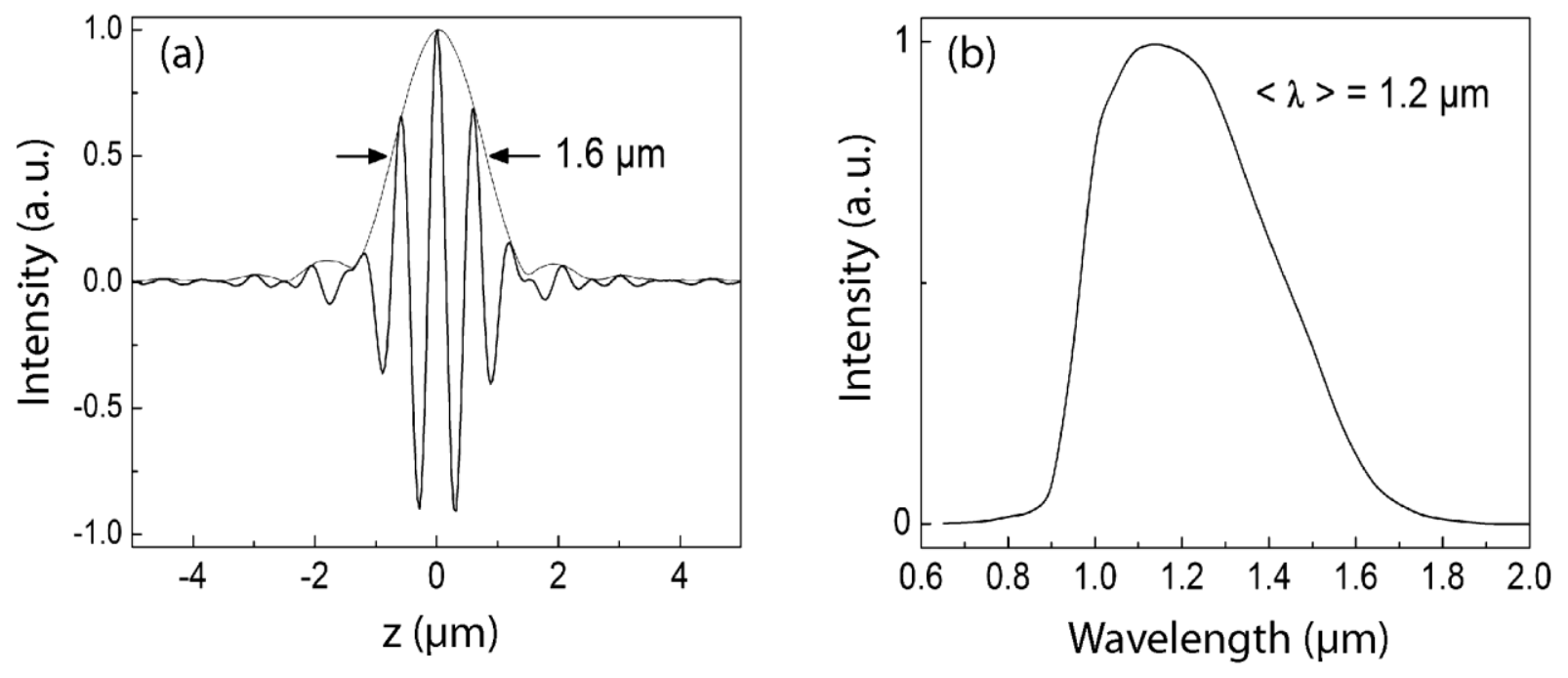

Fig. 4 


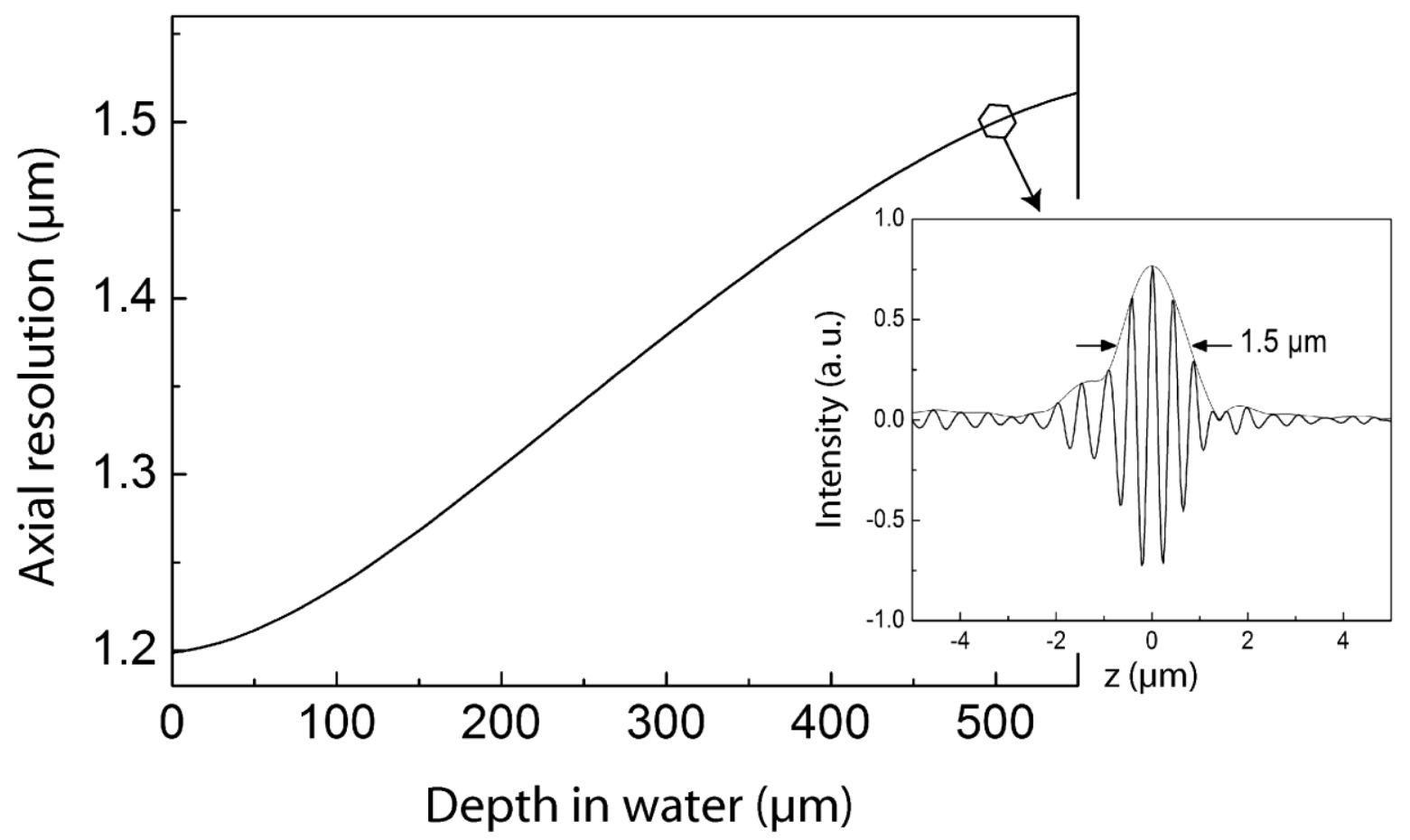

Fig. 5 


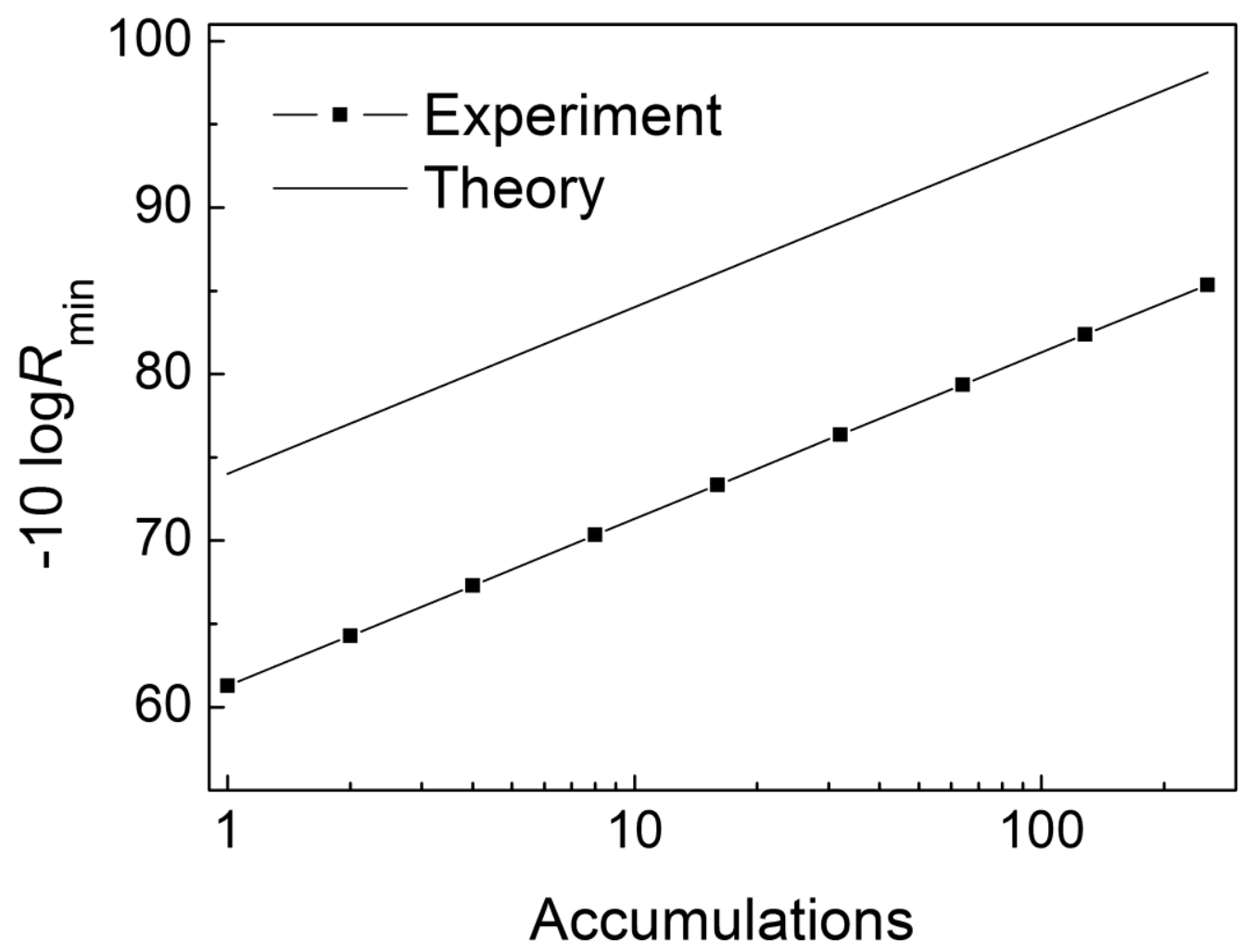

Fig. 6 


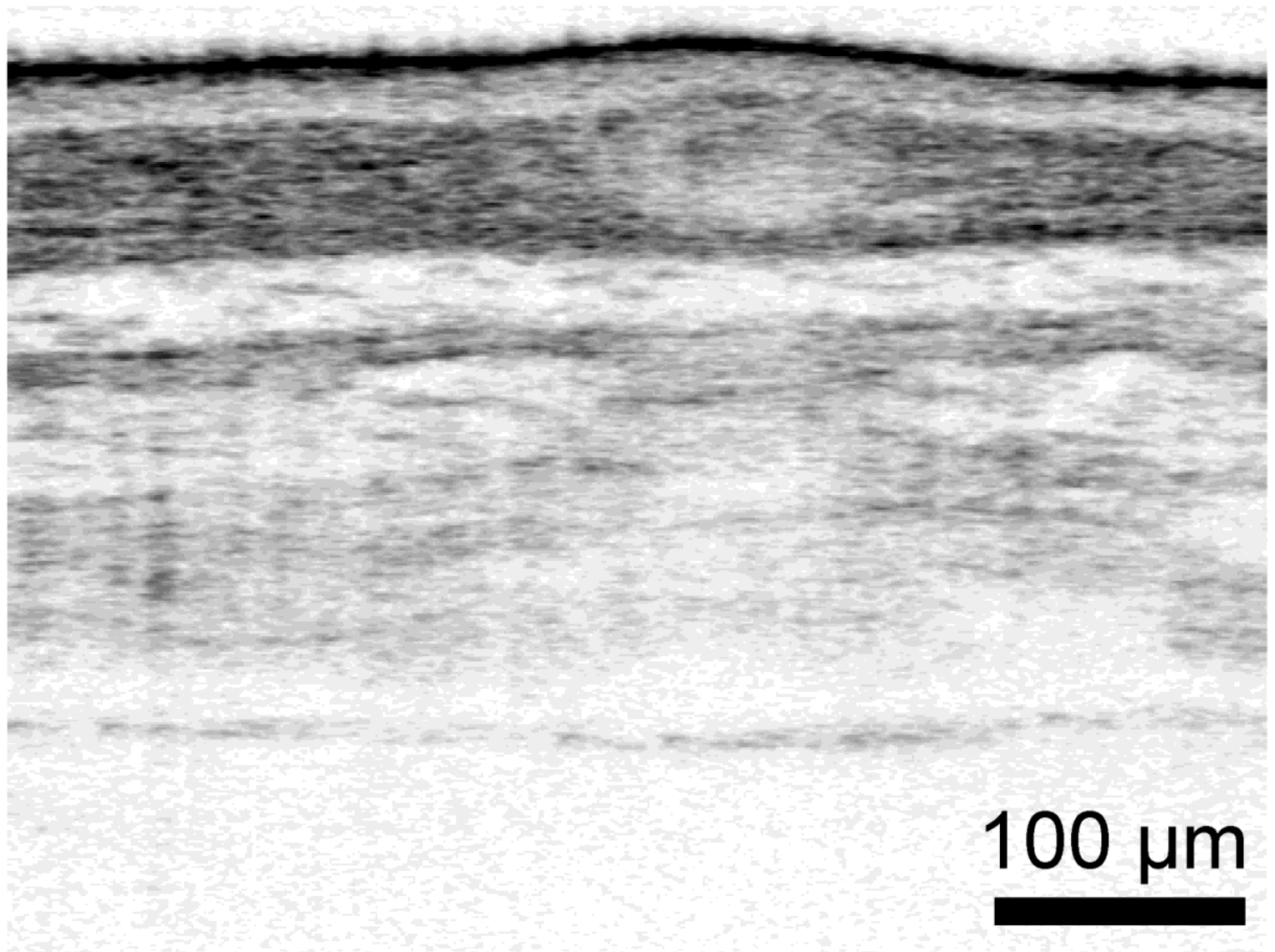

Fig. 7 